\title{
54 \\ DDS: The Data Delivery System
}

\author{
Andres F.(1) (2), Boulos J. (2) \\ (1) visiting researcher at NACSIS R\&D Center 3-29-1 Otsuka, Bunkyo-ku Tokyo 112 Japan \\ Tel: (81) 3-3942-5940 Fax: (81) 3-5395-7064 andres@ rd.nacsis.ac.jp \\ (2) member of IFATEC/Euriware, 12-14 rue du Fort de St-Cyr Montigny Le Bretonneux \\ 78067 St Quentin-en-Yvelines France Tel: (33) 1-3014-5444 Fax: (33) 1-3014-5416 \\ boulos@club-internet.fr
}

\begin{abstract}
DSS is a plug-ins based data delivery system extension of the application-oriented DBMS Phasme. It is intended to be used as a high performance customizable plug-ins backend for widely varying application domains. It is designed to satisfy maximum of application requirements and information systems' needs and to get maximum database performance out of today's hardware trends. The architecture has already achieved success in supporting video processing (Andres, 1996a, 1996b) and work is well under way in a project where it is used as a hypermedia server. DSS provides an extensible layer supporting plug-ins mechanisms and employs both inter and intra-operation parallelism. In this paper, we describe how we tackled the data delivery functionality with our open system.
\end{abstract}

\section{Keywords}

Application-oriented database system, parallel processing, plug-ins management

\section{INTRODUCTION}

The Decision Support System (DSS) architectures have evolved from the mainframe-based, proprietary application developments to the generation of open, distributed architectures, integrating client-resident query tools with server-based relational databases. The next generation of DBMSs will be confronted to a large variety of machines belonging to wider heterogeneous networks. Users as DSS analysts' requirements are changing to get better and faster accesses to data. Besides, data are characterized by a wide set of datatypes. Information system developers require data warehouse scalability, the ability to leverage corporate and industry investments in relational technology, minimum application redevelopment as the data 
model is modified, and also "plugs and play" support a variety of performance-optimized data models according to the target application.

Furthermore, developers use more and more personal workstation with larger and larger main memories. Main memory of $256 \mathrm{MB}$ are already affordable and CPUs can perform at 50 MIPS. The results published (KIM, 1994) indicate room for further improvement and a need for more effective application support in the field of data delivery.

The current commercial DBMS technologies (RDBMS, OODBMS, DOODMS) force the customers to adapt their needs and requirements due to the lacks and shortcomings of these technologies. Fully open database systems are required to overcome these shortcomings.

This paper describes how we tackled the Data Delivery functionality with our system called DDS (Data Delivery Systems) that was developed on the top of Phasme (Andres, 1995).

Phasme is a novel application-oriented DBMS (AODBMS) developed to provide a highly customizable parallel client/server DBMS to satisfy both hardware and customer trends.

By combining previous experiences in parallel database system design (EDS ESPRIT II project, Chameleon Project), in query optimization and benchmarking (Pythagoras ESPRIT III project), and also in information system architecture, DDS has been developed with the following specific design objectives:

1. Customizability: The architecture of DDS integrates the concept of many-sorted algebra. As this system is going to be used as the back end for applications in widely varying domains, it has to provide type-extensibility but also there is a need to satisfy new application requirements. DDS design has been done to support active, object-oriented, relational and deductive data models. The different data models are supported via user front ends. A major benefit of this approach is the speed of query processing and the adaptability to reach customer requirements through a vertical customizability.

2. Efficiency: The novelty of DDS plug-ins enables efficient manipulations of Phasme data structure. Vertical plug-ins mechanisms from the data type definition to the query execution level fully use the power of Phasme.

3. Scalability: In the context of information systems with large sets of data, DDS design exploits three strong technologies of Phasme architecture:

- Distributed shared virtual memory.

- Memory mapped file.

- Distributed servers.

We remind that the memory manager of Phasme follows a main memory-based design.

4. Parallelism:

The DDS system exploits both shared-store and all-cache architectures. The system can be tuned either to use only inter-operator parallelism, or to use both inter-operator and intraoperator parallelisms according to the DDS plug-ins. The first approach allows browsing query optimization to reuse query results. The algebraic operators are the units for parallel execution. Their result is completely materialized. The second approach enables to use deeper parallel capabilities of the target environment.

In this paper, we concentrate on the architecture of DDS and report on performance experiments. The remainder of the paper is organized as follows. Section 2 describes the overall of the architecture. Section 3 reports on performance modeling and experiments made with the prototype implemented on a SPARCstation 20. Section 4 concludes. 


\section{ARCHITECTURE OVERVIEW}

The architecture of DDS stemed first from the teachings of previous DBMS prototypes (Bergsten 1991, Singhal 1992, Jagadish 1994, White 1994, Boncz 1995) and second from the decision to use strong state-of-the-art technologies which have already been thoroughly tested.

DDS is a system which enables information designers to adapt the kernel according to the application requirements. The customization of the Phasme kernel is done vertically from the data type to the execution model according to the data delivery interface language. It follows the manysorted algebra concept (Guting 1993). It is an answer to support efficiently large range of application domains. Each application provides its own data semantic (e.g. relational, object) to the database kernel. It also imposes its specific data structures and indexes, its query optimization strategies and execution models as it is shown in Figure 1. This enables the improvement inside the DBMS kernel of both the functional behavior and the performance behavior of the application.

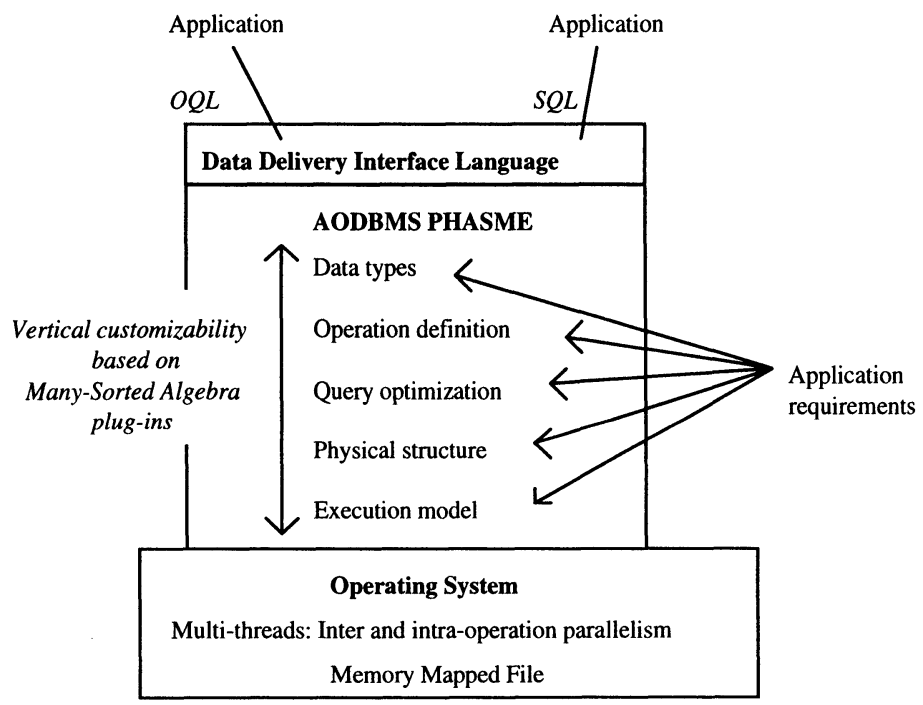

Figure 1 DDS architecture

An opened database system as the data repository of the information system is a solution to adapt the data management specifically to each application. As it has been pointed out in (Gray 1992), the physical data independence is also one of the cornerstones of the new generation of DBMSs. The data structure provided by Phasme is called Extended Binary Graph (EBG). As it is shown in Figure 2, this data structure combines three strong concepts: the DBGraph approach (Thevenin 1989) (ref 1), the fully Decomposed Storage Model (Valduriez, 1986) (ref 2) and the Graph Data Model (Kunii, 1990) (ref3). The EBGraph is based on no oriented arcs, a set of arcs representing 
one object item. Each arc is composed by two extremities (OID, VALUE) which can be inversed according to access methods. Each value is stored only once.

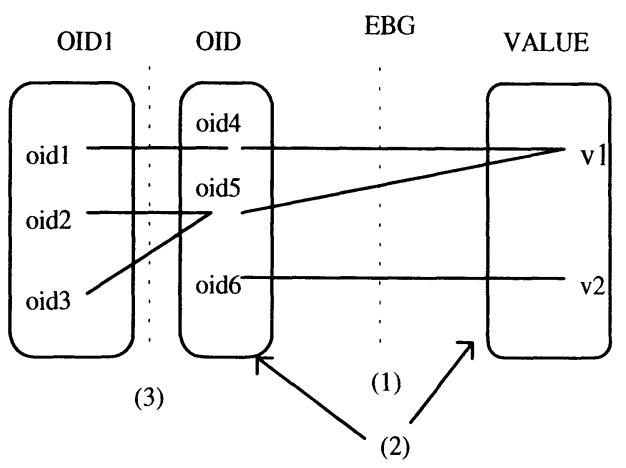

Figure 2 Phasme Data structure

The pointer orientation of the data structure is the key feature to get high performance and a customizable data storage to support various access methods as indexes (Lehman 1986) or Bang files (Freeston 1986, 1987). The database consists of a number of EBG memory mapped files which are Unix Files. The formats of the data on disks and in memory are the same to provide efficient data access. The data manager use no pointer swizzling mechanism in order to map data into memory. One drawback could be a lower reliability to access persistent objects by no deferencing standard virtual memory pointers, introducing the need for software checks. The use of no pointer swizzling mechanism to support Extended Binary Graph is a challenge to achieve high performance.

\section{DDS PERFOMANCE EVALUATION}

In this section, we report early performance experiments with the DDS prototype. We concentrate on the performance of the customized kernel layer. The platform used in the result presented here is a bi-processor Sun SPARCStation 20/50 Mhz running Solaris 2.4, with 96 MB main memory, $16 \mathrm{~KB}$ data-, $20 \mathrm{~KB}$ instruction and $1 \mathrm{MB}$ secondary cache, $1 \mathrm{~Gb}$ local disk and swap space.

In order to get insights on the behavior of DDS, we have experimented a subset of the $\mathrm{OO} 7$ Benchmark (Carey 1993) in increasing order of complexity: exact lookup (Q1), scan (Q2, Q3, $\mathrm{Q7)}$, path lookup (Q4), single-level make(Q5), join(Q8), insert and delete.

The metric used in the performance experiments is the completion time. The experimental database was generated automatically following the specification of the 007 Benchmark. The size of the medium database (fanout 9) is equal to $69 \mathrm{MB}$.

The parallelization of the operation relies on the Solaris thread management system. Solaris System effectively distributed the work between the available processors, when several threads 
work concurrently. We assume a perfect parallelism resulting from a good repartition between threads and processors.

The performance results of the queries for the medium/9 database are shown below in Table 1 .

Table 1 Performance

\begin{tabular}{lll}
\hline Queries & Cold Time & Hot Time \\
\hline Q1 & $0.67 \mathrm{~s}$ & $0.46 \mathrm{~s}$ \\
Q2 $(1 \%)$ & $0.52 \mathrm{~s}$ & $0.51 \mathrm{~s}$ \\
Q3 $(10 \%)$ & $0.57 \mathrm{~s}$ & $0.53 \mathrm{~s}$ \\
Q4 & $0.74 \mathrm{~s}$ & $1.56 \mathrm{~s}$ \\
Q5 & $1.7 \mathrm{~s}$ & $1.56 \mathrm{~s}$ \\
Q7 $(100 \%)$ & $1.8 \mathrm{~s}$ & $1.62 \mathrm{~s}$ \\
\hline
\end{tabular}

Figure 3 compares the results of the cold and hot exact match lookup query execution varying the number of threads for the medium database (fanout 9). In each case, the system used the clustered index to provide high performance.

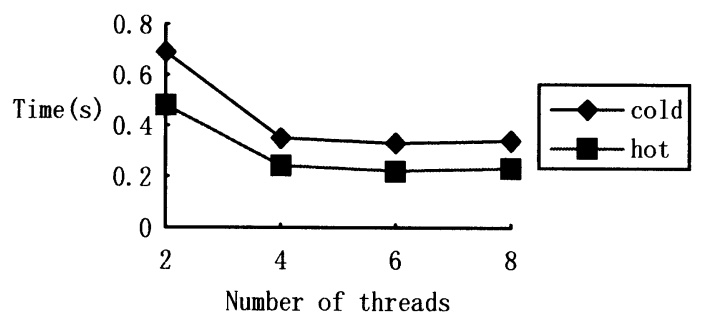

Figure 3 exact match lookup query

Figure 4 compares the results of the cold and hot scan execution of the all atomic parts varying the number of threads for the medium database (fanout 9). The degree of intra-operator parallelism enables to improve the efficiency of the $\mathrm{OO}$ query processing.

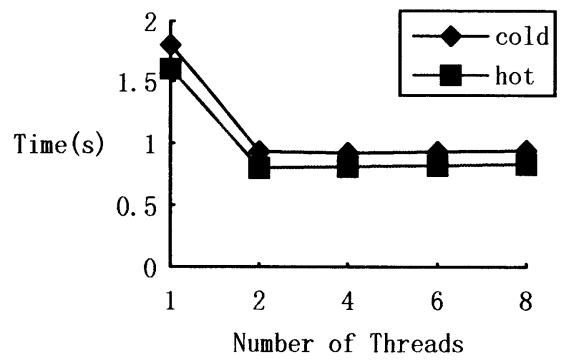

Figure 4 scan query, medium $\mathrm{db} / 9$ 
It is also important to evaluate the performance behavior of structural operations as insert and delete to stress build ability. Figure 5 shows the results of insert and delete operations. The intra-operation parallelism between the insertion operations decreases the response time by a factor 5 if the number of threads is equal to the number of new composite parts. The same result is obtained for the delete query.

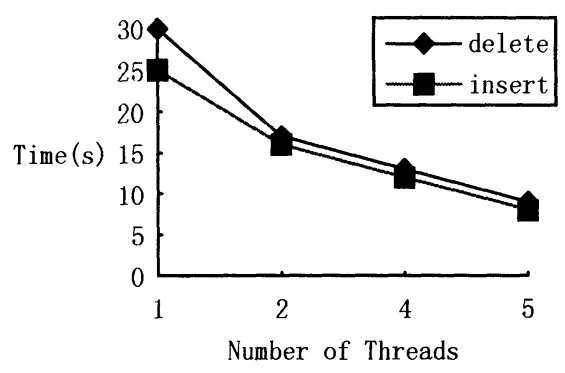

Figure 5 delete and insert, cold, medium $\mathrm{db} / 9$

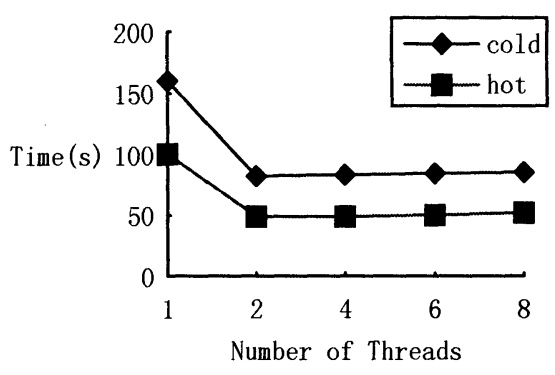

Figure 6 traversal T1, medium size

Raw traversal speed has been also studied. Figure 6 shows that the cold times are maximal for a factor 1.6 slower than the hot times. The main memory approach is not a major cost factor. The association between EBG and parallelism query execution is the key factor to provide high performance. Partition in different memory mapped files is also a performance improvement in terms of data locality and caching.

\section{CONCLUSION}

DDS is an extension of the parallel Application-Oriented DBMS whose goal is first to provide a new approach for information processing technology and second to satisfy both the requirements of the new generation of information systems and the hardware trends. DDS plug-ins enables to use efficiently the main-memory data storage structure of Phasme called Extended Binary Graph (EBG).

In this paper, we have presented the architecture of DDS, and reported experiments with the current prototype. We focused on applying DDS on OO operations. We consider the following features to be the most significant contributions of the DDS project:

- Plug-ins for parallel query processing. DDS enables to implement plug-ins supported by the parallel dataflow execution model of Phasme. The plug-ins concept provides the data model independence but it allows to cooperate with any. Compared to other database systems such Texas and QuickStore, this leads to support efficiently intra-operation parallelism for object management.

- Run-time system. The run-time exploits the virtual memory functions and advanced features of major operation systems (e.g. SOLARIS, IRIX, WIN NT) to minimize the overhead of the 
parallelism. Furthermore, it implements a data storage manager which doesn't use pointer swizzling to map data into memory. The data format on disk is the same as in memory.

The early implementation of the DDS prototype together with performance measurements of a subset of an object benchmark was useful to point out two things. First, the implementation of an Application-oriented DBMS based on memory mapped files and allowing intra-operation and inter-operation parallelisms improves the database management performance. Second, the customizability of the DDS implementation allows to reach the applications' needs with higher simplicity.

This performance experiment using the 007 benchmark has been done to study response time for single-user queries. It shows a good parallel query processing. It also partly confirms our intuition about the need of a new generation of data storage kernel. Future experiments are planned to study the behavior of multimedia applications using Phasme.

\section{REFERENCES}

Andres, F. and Boulos, J. (1995) Phasme: A Parallel Application - Oriented Database System, Ifatec technical report No 950120.

Andres, F. Ihara, K. Boulos, J. Ono, K. and Yasuhiko Y. (1996a) The OLVP (Online Video Processing) System Architecture based on Distributed VOD Servers in Proc. DMS 96, Hong Kong.

Andres, F. Ihara, K. Boulos, J. Ono, K. and Yasuhiko Y. (1996b) Performance evaluation of the OLVP (Online Video Processing) System in proceeding COMPSAC 96, Coreen, Seoul.

Bergsten, B. Couprie, M. and Valduriez, P. (1991) Prototyping DBS3, a Shared Memory Parallel Database System in Proc. PDIS.

Boncz, P. A. and Kersten, M. L. (1995) Monet: A impressionist sketch of an advanced database system" In Proc. IEEE BITWIT workshop, San Sebastian (Spain).

Carey, M. and DeWitt, D.J. and Naughton, J.F. (1993) The Dec OO7 Benchmark in Proc. ACM SIGMOD.

Freeston, M. (1987) The Bang file: a new kind of Grid File in Proc of ACM SIGMOD.

Freeston, M. (1986) Data Structures for Knowledge Bases: Multi-Dimensional File Organisations, ECRC. Technical Report TR-KB-13.

Guting R.H. (1993) Second Order Signature: A tool for specifying data models, query processing and optimisation in proceedings ACM-SIGMOD, Washington.

Gray J., Reuter A. (1992) Transaction Processing: Concepts and Techniques. Morgan Kaufman.

Jagadish, H. Lieuwen, D. Rastogi, R. and Silberschatz, A. (1994) Dali: A High Performance Main Memory Storage Manager in Proc. of the 20th Int. Conference on VLDB, Santiago, Chile, September 12-15, pp 48-59.

Kim W. (1994) Modern Database Systems. Addison-Wesley, ACM press.

Kunii, H.S. (1990) Graph Data Model and its Data Language., Springer-Verlag, Tokyo.

Lehman, T.J. and Carey, M.J. (1986) A Study of Index Structures for Main Memory Database Management Systems" in Proc. 12th Conf. on VLDB, Kyoto, Japan. 
Thevenin J. M. (1989) Architecture d'un Systeme de Gestion de Bases de Donnees Grande Memoire, PhD Thesis of Paris VI University.

Singhal, V. Kakkad, S. V. and Wilson, P. R. (1992) Texas: An Efficient, Portable Persistent Store in Proc. 5th Workshop on Persistent Object Systems, September pp 11-33.

Valduriez, P. and Khoshafian, S. and Copeland, G. (1986) Implementation techniques of complex objects, in Proc. of the Int. Conf. on VLDB, Kyoto, Japan.

White, S. J. and DeWitt, D. J. (1994) QuickStore: A High Performance Mapped Object Store in Proc. of the ACM SIGMOD, Meneapolis, MN.

\section{BIOGRAPHY}

Dr. Frederic Andres is a specialist in database system, query processing, and distributed information systems. He worked at Bull on the system DBS3 in the EDS project II project and on query performance tools in the Pythagoras Esprit III project. He joined Ifatec/Euriware company after gaining his European PhD from University of Paris VI. Inside International $\mathrm{R} \& \mathrm{D}$ project, he is currently holding a visiting position at NACSIS (Japan). He was also been consultant in major projects (Alma project, Bibliotheque de France, French budget ministery). He currently leads the Chameleon and the DDS project to achieve a new generation of information systems that embodies novative ideas on the way to combine high performance query processing and information system's requirement. He is co-author of the Binary Document Manipulation Benchmark to stress the effectiveness of the RDBMSs to manipulate binary documents (e.g. OL2 documents).

Jihad Boulos is a $\mathrm{PhD}$ student in computer science at Paris VI University and a researcher at Ifatec computer company. His main research work focusses on performance evaluation of database systems. Analytical models, simulation, and benchmarking are the main subjects of interests; in addition, integration of neural network techniques in database performance prediction processing and query optimisation is a part of on-going research action. Jihad Boulos got a B.S. in computer science from the American Lebanese University in Lebanon in 1991, a Master degree from the University of Paris VI in 1993, and the date of the doctoral defense is fixed at the end of July 1996. 